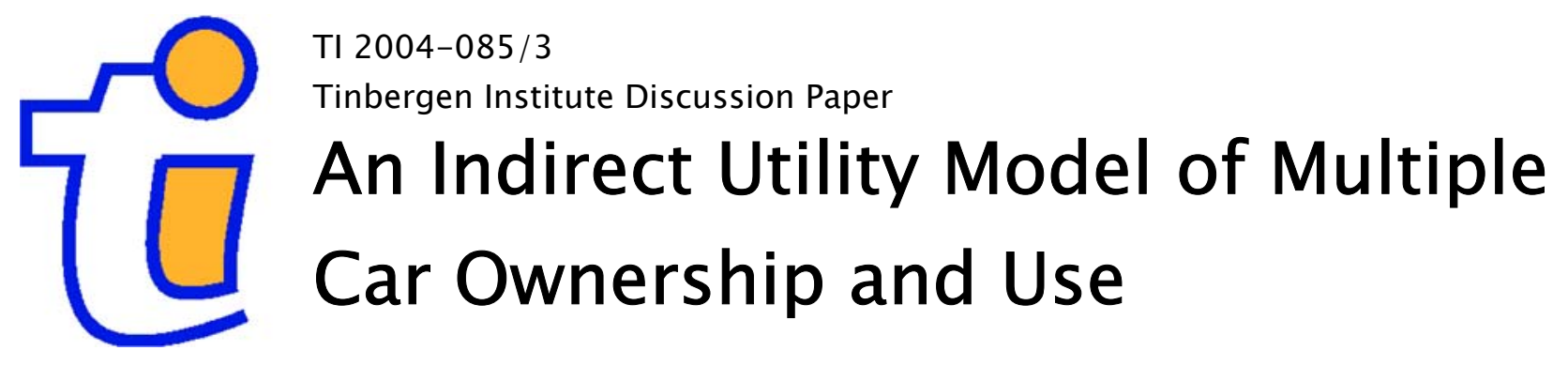

Jan Rouwendall
John Pommer

${ }^{\prime}$ Faculty of Economics and Business Administration, Vrije Universiteit Amsterdam, and Tinbergen Institute;

2 Connekt, Delft. 


\section{Tinbergen Institute}

The Tinbergen Institute is the institute for economic research of the Erasmus Universiteit Rotterdam, Universiteit van Amsterdam, and Vrije Universiteit Amsterdam.

Tinbergen Institute Amsterdam

Roetersstraat 31

1018 WB Amsterdam

The Netherlands

Tel.: $\quad+31(0) 205513500$

Fax: $\quad+31(0) 205513555$

Tinbergen Institute Rotterdam

Burg. Oudlaan 50

3062 PA Rotterdam

The Netherlands

Tel.: $\quad+31(0) 104088900$

Fax: $\quad+31(0) 104089031$

Please send questions and/or remarks of nonscientific nature to driessen@tinbergen.nl.

Most TI discussion papers can be downloaded at http://www.tinbergen.nl. 


\title{
An indirect utility model of multiple car ownership and use
}

\author{
Jan Rouwendal ${ }^{1}$ and John Pommer ${ }^{2}$ \\ Department of Spatial Economics \\ Free University \\ De Boelelaan 1105 \\ $1081 \mathrm{HV}$ Amsterdam \\ The Netherlands \\ Tel. 31-20-4446093 \\ Fax: 31-20-4446004 \\ E-mail:jrouwendal@feweb.vu.nl
}

This version: January 4, 2004

Keywords: automobile demand, multiple car ownership, demand for car kilometers JEL Classification Codes: R41, D13

\begin{abstract}
The number of households with two or more cars is steadily increasing over time. This has potentially significant implications for the reaction of car use and ownership to changes in fuel prices and fixed car costs. In this paper, we develop a model that is based on the idea that limited substitutability between desired car use of different household members is the driving force behind multiple-car ownership. We develop a model structure that is consistent with economic theory and show that it can be used to extend an existing model for ownership and use of a single car to multiple car ownership and use. The extended model is estimated on Dutch data concerning households with one or two cars. An application to the effects of shifting the burden of car taxes from fixed to variable costs is provided.
\end{abstract}

\footnotetext{
${ }^{1}$ Jan Rouwendal is also at the department of Social Sciences of Wageningen University. He is affiliated to the Tinbergen Institute, Amsterdam.

${ }^{2}$ John Pommer is also at Connekt, Delft.
} 



\section{Introduction}

The automobile is one of the most important consumption goods in modern societies. It may even be said that widespread automobile ownership is one of the defining characteristics of these societies. Participation in social life is difficult for those who do not own a car, and it is therefore not surprising that car ownership has been steadily increasing over time. For instance, in the Netherlands the share of households not owning a car has been decreasing for decades, and more recently that of households owning just one car has also started to decrease. ${ }^{1}$ The increasing share of multiple car households has potentially important consequences for the reaction to changes in fixed and variable costs of cars. When fuel prices increase, multiple car households can change the distribution of household car kilometers towards the vehicle with the lowest fuel costs per kilometer, whereas single car households can only realize such an effect by replacing the car. When car prices, or fixed costs associated with car ownership, increase, it is easier for multiple car households to decrease the size of their car park than it is for single car households. It may therefore be conjectured that the increasing share of multiple car households has consequences for the reactions to both types of cost changes. In this paper we develop an economic model that allows us to study this phenomenon.

Economic theory suggests that decisions with respect to car ownership and use are based on a single set of preferences. Decisions made with respect to the number of cars to be owned and the numbers of kilometers to be driven with them should therefore be derived from the maximization of the same utility function under the appropriate budget constraint. Application of this straightforward reasoning in empirical work is complicated by the fact that cars have discrete (ownership) as well as continuous (use) aspects to consumers. The fixed cost (depreciation, etc.) can be interpreted as a kind of entrance fee that allows a household to consume as many car kilometers as it likes at the variable costs associated with the car. Households decide to own a car only when the benefits associated with car use exceed the fixed cost. This reasoning was elaborated into an empirical model for ownership and use of a single car by De Jong (1991) and the model structure we develop in this paper enables us to generalize it to multiple car ownership and use.

The analysis of ownership and use decisions of multiple car households within a theoretically consistent framework is demanding. The use of elementary discrete choice models, which have been popular in the study of car type choices since their development in the 1970s, is ruled out by Roy's identity, which relates the utility of owning a car to the number of kilometers driven by it. The linearity of the utility functions conventionally used in these models would imply a demand for kilometers that is insensitive to price and income. Even though Train (1980) proposed a partial solution to this problem by including commuting trips in a nested model structure, in general a discrete choice model with a nonlinear utility function is required for a theoretically consistent model of ownership and use. Mannering and Winston (1985) provided one of the first analyses of automobile demand that used such a model.

Another requirement implied by the theory is that the utility of not owning a car should be related to that owning one. The utility of not owning a car follows from restricting the number of kilometers driven with that car to zero and removing the fixed costs from the budget restriction. Alternatively, one could set the (virtual) price of car use equal to the reservation value in the indirect utility value. De Jong (1990) seems to have been the first to give a rigorous treatment of

\footnotetext{
${ }^{1}$ For instance, in the Netherlands the share of households owning one car was stable at $60 \%$ between 1990 and 1996 , but has steadily decreased to $55 \%$ since then. The share of households owning two or more cars has increased from $13.3 \%$ in 1991 to over 20\% (Source: CBS Statline).
} 
this issue in an empirical model. His investigation is restricted to single car households and, therefore, to the choice between owning 0 or 1 car. As far as we are aware, there have been no generalizations of this approach to households with multiple cars, with the exception of De Jong (1991) to be discussed later in this paper. ${ }^{2}$

Such a generalization requires a framework for analysis in which a household can have at least two cars, while the kilometers driven by these cars are imperfect substitutes. This implies that, in general, we cannot deal with the total number of kilometers driven by household members as a single commodity. The behavior of households with a single car can be analyzed by imposing the restriction that the number of kilometers driven by one car is equal to 0 , and that of households without a car by setting both numbers of kilometers equal to 0 . Such a framework is necessary for a theory-consistent analysis of the behavior of multiple car households, but it is not easy to implement. For instance, Mannering and Winston (1985) deals with households owning one or two cars. The indirect utility function and the car use equation they estimate for households with two cars are analogous to that of single car households. This means that they specify the indirect utility of owning two cars to be the same function as that of owning two cars, while allowing the parameters to be different. Economic theory suggests that the specifications of the indirect utility functions referring to ownership of one and two cars should be different, while the same parameters should occur in both. The theory requires that the utility of owning one car can be derived from that referring to ownership of two cars by restricting the number of kilometers driven by one of the cars to 0 and taking into account that there are fixed cost of one car only. The model to be derived in the present paper follows this approach.

Even though the problems that are encountered when developing a structural model for ownership of multiple cars are substantial, this did, of course, not mean that multiple car ownership and related issues have not been studied. Rather, it implied that some of the rigor of economic theory had to be given up in order to arrive at a tractable model formulation. Many interesting findings were reported in the literature on the basis of such studies. For instance, Mannering and Winston (1985) report that the demand for automobile kilometers of households with two cars is less sensitive to changes in operating costs than that of single car households. They attribute this, quite plausibly, to the possibility to substitute between the two cars, even though their model does not explicitly incorporate such behavior. Mannering and Train (1985) provide a fine verbal discussion of a number of aspects that are relevant in studying households with two or more vehicles. For instance, they deal with the relation between the number and type of autos owned and the relation between the numbers of miles traveled on each vehicle in multivehicle households. There is an enormous amount of literature on multiple car ownership that uses a reduced form approach or models that are conditioned on some aspects (such as the number of cars). We will not attempt a review here, but instead discuss a few of topics that are relevant for the purposes of the present paper.

Our intention to concentrate on some specific aspects of multiple car ownership implies that we pay less attention to some other aspects of the demand for cars. In particular, one aspect that has been dealt with extensively in the literature, but will receive less emphasis in the present paper is the choice of the car type. See, for instance, Train and Lohrer (1983) that later appeared as a chapter in Train $(1986)^{3}$ for a discrete choice model dealing with car type choice of households with one and two cars. This model does not deal with car use and is therefore less suitable to

\footnotetext{
${ }^{2}$ See footnote 10.

${ }^{3}$ Other references are provided in Mannering and Train (1985).
} 
study the interaction between car use and ownership. ${ }^{4}$ The model of the present paper will focus on this interaction, but pays only limited attention to type choice.

There are a number of studies that consider car use in multiple car households while taking the number of cars as given. Early examples are Mannering (1983) who considered two linear simultaneous equations referring to the use of the two cars in a household, and Hensher (1985) who uses a more extensive system of equations referring also to fuel cost per kilometer and fuel efficiency. Mannering stresses the potential importance of substitution towards the more efficient vehicle in response to fuel price changes, while Hensher's position is nuanced by the larger amount of details present in his model. More recently, Golob and co-authors (see Golob, Kim and Ren, 1996; Golob and McNally, 1997) used systems of linear equations in order to study activity participation and car use by household members. One of these studies observes that the higher operating cost of one vehicle results in a shift in usage to the other. (Golob, Kim and Ren, 1996, p. 116). An important suggestion emerging from this literature is that car use within the household is embedded within the pattern of household activities. Ownership and use of the first and second car should therefore not be regarded as separate issues, but as interrelated.

It is the purpose of the present paper to develop and estimate a model of car ownership and use in multiple car households that is consistent with economic theory. An obvious advantage of such a structural model is the closer relation with economic theory and the operational model. A related aspect is the possibility to interpret the parameters as elements of the household's utility function, which enhances transparency and eases interpretation. A single set of parameters referring to the utility function explains all observed behavior with respect to ownership and use. This makes the model parsimonious. Finally, these features of the model may increase its suitability for policy simulations.

In the next section we develop the general model structure for ownership and use of cars that is suggested by standard microeconomic theory. We proceed there as follows. The kilometers driven by different cars appear as separate commodities in the household utility function. In order to consume these commodities, the household must own cars, which is costly. These costs can be overcome by the benefits derived from car use. A car will therefore only be owned if the benefits of using it exceed its fixed cost. We impose additional structure on the model by requiring that the variable costs of the cars owned by the household enter indirect utility through a variable that can be interpreted as a composite price of car mobility. In the special case in which the kilometers driven by these cars are perfect substitutes, the composite price is equal to the lowest of the variable costs of the separate cars and only the cheapest car will be used. This means that the household will never use more than one car, and therefore will never own more than one car. ${ }^{5}$ In the more likely case where car kilometers are imperfect substitutes, there is something to be gained by owning two cars instead of one. Imperfect substitutability occurs as soon as two household members would like to use a car at the same time for different trips. An obvious example is a dual-earner household with the two workers employed at different locations, and both would like to use the car for commuting purposes. Even though in this situation a single car can be used when one worker brings the other to work in the morning and picks him or her up in

\footnotetext{
${ }^{4}$ Rouwendal and de Vries (1999) who study the choice of fuel type with a discrete-continuous model find a significant effect of the variable cost on the demand for kilometers.

${ }^{5}$ When the costs of the two cars are equal, the distribution of the car kilometers over the cars is a matter of indifference. The household may therefore as well allocate all these kilometers to one car and save the fixed cost of the other.
} 
the evening, it is usually much more convenient to have two cars available. The gain in utility associated with ownership of two cars appears in the model as a lower price for the composite good. This gain is traded off against the higher fixed costs associated with owning two cars. The remainder of the paper is organized as follows. In Section 3 we discuss the data set we use in the empirical work and perform some exploratory analyses. Section 4 discusses some estimation issues, and reports estimation results. Section 5 provides further discussion of these results and a simulation of the effects of shifting the burden of car taxes from fixed to variable car costs. Section 6 concludes.

\section{An economic model for multiple car ownership}

\subsection{Introduction}

In this section, we develop our model of a household that has to decide on the number of cars to be owned and their use. We start in subsection 2.2 with a general model in which the number of cars is fixed. This is basically the standard model of a utility-maximizing consumer, as described, for instance, in Deaton and Muellbauer (1980). The only specific feature that has been added is that the consumer has to pay a fixed cost in order to be able to consume car kilometers.

The fixed number of cars should be interpreted as the maximum number that can be owned by the household. The choice of this number is not crucial for our results as long as it is set at a sufficiently high value, as will be explained.

This choice of the actual number of cars is introduced in subsection 2.3 by adding a constraint on the number of cars to the basic model. This constraint determines the actual number of cars owned by the household and should be interpreted as self-imposed. The fact that one or more potentially available cars cannot be used will decrease (or at least not increase) utility, but since no fixed costs have to be paid for these cars, the net effect of the restriction on utility may well be positive. The optimal number of cars is that for which utility is maximal.

In subsection 2.4 we introduce the notion of indirect separability. It means that the variable costs of the various cars enter the indirect utility function only via a function that will be interpreted as a composite price of car use. Rationing the number of cars owned by the household increases the composite price of car mobility, and this increase indicates the cost of limiting the number of cars. The composite price function determines the distribution of the total number of kilometers driven by the household over the cars it owns. The possibilities to substitute between use of the various cars caused by differences in variable costs are therefore also determined by this function. Subsection 2.5 discusses the introduction of differences in car quality into the model. Although quality choice is not a central topic of the present paper, we have to take it into account in order deal with the substitution between the use of cars within the household that occurs in reaction to differences in variable costs. Such differences reflect differences in other car characteristics, which we summarize as 'quality'. We outline a model in which higher quality can be obtained at the expense of higher fixed and variable costs.

Finally, we propose a specification of the function describing the composite price of car use in subsection 2.6 and discuss its implications in subsection 2.7. It is used there to generalize de Jong's (1991) indirect utility model describing the choice between no car and a single car to cases in which an arbitrary number of cars can be owned. This specification is used in the empirical section. 


\subsection{A basic model}

In our basic model, all cars are identical: they have the same fixed and variable costs and other characteristics. The model concerns a household that derives utility from the kilometers driven by $N$ such identical cars and a composite consumption good. $N$ is the predetermined maximum number of cars that can be owned by the household. $N$ can be arbitrarily large, but, in practice, there are few households that own more than four cars. The amount consumed of the composite good is denoted as $q$, and the numbers of car kilometers driven as $x$, with $x$ an $N$-dimensional vector. The utility function is:

$u=u(q, x)$.

Utility is maximized subject to a budget constraint:

$$
\begin{aligned}
p x+q & =y-k N \\
& =y^{\prime},
\end{aligned}
$$

where $p$ denotes the $N$-dimensional vector of variable costs of the cars. The price of the composite good is used as the numéraire. The right-hand side of this equation gives the household's disposable income $y^{\prime}$, which is defined as the "difference between household income $y$, and the fixed costs of the cars owned by the household." These costs are indicated as $k N$, with $k$ the fixed costs per car.

Maximization of utility subject to this budget constraint leads to demand equations for the car kilometers and the composite good. The utility experienced by the household is given by the indirect utility function $v$ :

$v=v\left(y^{\prime}, p\right)$.

The model developed thus far takes the number of cars as given, and therefore cannot be used to study the choice of the number of cars. We consider that extension in the next subsection, but note here that the model as developed thus far can be used to study the use of different cars in the household at a very general level. Even though we have assumed that all cars are identical, the use of the various cars owned by a household may well be different since we have not assumed that the utility function $u$ is symmetric in the numbers of kilometers driven by the various cars. The marginal utility functions of the kilometers driven by different cars may therefore differ, and so may the optimum numbers of kilometers. For instance, one car may be used for commuting and another one for social purposes, shopping and holidays. This generality is a consequence of our treatment of the kilometers driven by different cars as separate commodities. Thus the kilometers driven by various cars may be substitutes as well as complements, the kilometers driven by one car may be an inferior good, whereas those driven by another car are normal, et cetera.

It seems reasonable to expect that a household will not use all its cars intensively if their number is large. It may even be the case that some cars are not used at all. The possibility of zero consumption is also present in conventional models of consumer behavior and is important for dealing with, for instance, non-smokers. However, in the present context there is an additional element involved since abstaining from the use of a car opens the possibility to decrease the fixed costs associated with car ownership by making the actual number of cars smaller than $N$. The lower fixed cost increases the amount of money that can be spent on other goods, and this enables the household to reach a higher level of utility. It may therefore be in the interest of the household to restrict the actual number of cars to a value lower than $N$.

Because of the lower fixed cost associated with limited car ownership, the household will find it beneficial not to own the maximum number of cars if the predetermined value of $N$ is set large enough. The optimal number of cars will then always be smaller than $N$. If the maximum number 
of cars would be set at a higher level (e.g. $N+1$ ), the optimal number of cars will not change and for this reason the choice of $N$ is arbitrary as long as it is a sufficiently large number.

\subsection{Optimal choice of the number of cars}

The choice of the optimal number of cars will now be introduced into the model by adding a restriction on car use. This restriction says that the number of kilometers of some cars (viz. those not owned by the household) has to be equal to 0 . This means that, for these cars, the demand for car kilometers is rationed. If a household owns $n$ cars with $0 \leq n<N$, then $N$ - $n$ elements of $x$ will be equal to 0 .

Now consider a household whose car ownership is described by an $N$-dimensional vector $d$ with only 0 's and 1 's as elements, with the 1's referring to the owned cars and the 0 's to the others. The effect of rationing on the indirect utility of the household can be described by virtual prices for the kilometers of the cars not owned by the household. These virtual prices are the values of the variable costs that would induce the household to drive no kilometers with these cars, were they available. ${ }^{6}$ The virtual prices are a function of the other prices and of the household's disposable income (computed without the fixed costs of the cars that are not owned). They are always at least as high as the actual prices of the commodity concerned.

The relevant prices for car use of this household are then given by a vector $p^{\prime}(d)$, with variable costs $p$ on the positions of the owned cars, and virtual prices in the other positions. The virtual prices will, in general, be different and depend on the need for using these cars. Disposable income is now defined as $y^{\prime}=y-k n$ and indirect utility can be written as:

$v=v\left(y-k n, p^{\prime}(d)\right)$.

If the household would use all $N$ cars if they are owned (i.e. the demand for kilometers would be strictly positive for all cars), then the virtual prices of the cars not owned are higher than their variable costs. These higher values reflect the cost of the rationing. The benefit associated with the rationing is that disposable income is higher because of the lower fixed car costs. The net effect on utility of restricting the number of cars thus depends on the relative magnitude of both effects.

If the household would not use all $N$ cars, restricting the number of kilometers of these cars to 0 imposes no real restriction on household behavior. The virtual price is in this case equal to the actual price. This means that there is no cost (in terms of utility) involved in not-owning such a car. It is therefore always beneficial not to own such a car, because of the fixed costs involved. A final issue to be discussed in the present subsection concerns the choice of the cars to be abandoned. In the model developed above we can distinguish between households that own the same number of cars but are located at different positions of the vector $d$. However, in practice we cannot distinguish between households with different vectors $d$ but the same number of cars. For instance, if a household owns one car, it is impossible to know whether this car is in the first, second, .. or $N$-th position of $d$ and their utilities may differ. It is therefore useful to reformulate the model in such a way that we can refer to households owning $n$ cars without having to specify the positions of these cars in the vector $d$.

In order to do this, we introduce some additional notation. Let $D$ be the set of all $N$-dimensional vectors with only 0 's and 1's as elements, and $D(n)$ the subset of all vectors $d \in D$ with the number of ones equal to $n$ (that is: $d^{\prime} d=n$ ). The utility $v_{n}$ of owning $n$ cars is defined as 'the

\footnotetext{
${ }^{6}$ See Neary and Roberts (1980).
} 
maximum utility that can be reached by a household whose car ownership is described by an element of $D(n)$.' That is:

$v_{n}=\max _{d \in D(n)}\left\{v\left(y-k n, p^{\prime}(d)\right)\right\}, \quad n=0,1, \ldots, N$.

The utilities $v_{n}$ take into account the flexibility that a household has in assigning the available cars to the tasks or persons most in need of them.

The model can now be used to study the choice of the number of cars. The optimal number $n *$ is that for which utility is highest:

$n^{*}=\arg \max \left\{v_{i}, i=0,1, \ldots, N\right\}$.

The model that has been developed here is completely general, in that it does not use other assumptions than those conventionally made in economic analysis in order to derive demand functions for commodities under conditions of rationing. The household is assumed to react in an optimal way to restrictions on the number of cars. Such a reaction may involve bargaining between household members, and it is assumed that the outcome of this process can be described by a household utility function possessing all conventional properties.

\subsection{Indirect separability}

Even though the generality of the model developed above is a clear advantage, empirical work requires further specification. We will now introduce some additional structure on the indirect utility function that gives some guidance for specifying such an empirical model. We assume that the indirect utility function is separable in the costs of car kilometers. This means that variable car costs enter the indirect utility function $v$ via a function $P$. In other words, it says that we can replace the vector of variable costs $p$ in (3) by a scalar $P$, whose value is determined by these variable costs:

$v=v^{\prime}\left(y^{\prime}, P\right)$

and

$P=P\left(p^{\prime}(d), y^{\prime}\right)$

with $y^{\prime}=y-n k$. The function $P$, which may depend on disposable income, will be interpreted as composite price for car kilometers, and it must be increasing with the variable costs of all cars.

An advantage of this further specification of the indirect utility function is that it allows us to give an intuitive account of the costs associated with restricted car ownership. We have just seen that some or all of the variable costs are virtual prices when the actual number of cars is lower than the maximum number. This means that the actual price of car mobility is higher than it would be if the maximum number of cars were owned. This is because having less than the maximum possible number of cars at their disposal restricts the household members in their activities: for instance, by eliminating the possibility to carry out sequences of activities that would be possible if an additional car were available. The difference between the constrained and unconstrained values of $P$ thus gives a useful summary measure of the benefits of owning a larger number of cars. Another consequence of this specification is that it allows us to generalize de Jong's (1990) model to situations with two or more cars, as will be shown later in this section. The number of kilometers driven by car $i$ owned by the household can be derived from (7) by means of Roy's identity as: 


$$
x_{i}=-\frac{\frac{\partial v^{\prime}}{\partial P} \frac{\partial P}{\partial p_{i}}}{\frac{\partial v^{\prime}}{\partial y^{\prime}}+\frac{\partial v^{\prime}}{\partial P} \frac{\partial P}{\partial y^{\prime}}},
$$

where $P$ should be evaluated at $p^{r}$ and $z^{r}$. It is useful to consider car use by a household as described by the total number of kilometers driven and the share of each owned car in this total. The total number of car kilometers $X$ is equal to:

$$
X=\sum_{i=1}^{N} x_{i}=-\frac{\frac{\partial v^{\prime}}{\partial P} \sum_{i \in O} \frac{\partial P}{\partial p_{i}}}{\frac{\partial v^{\prime}}{\partial y^{\prime}}+\frac{\partial v^{\prime}}{\partial P} \frac{\partial P}{\partial y^{\prime}}},
$$

where $O$ denotes the set of cars owned by the household. The share $s_{i}\left(=x_{i} / X\right)$ of the $i$-th car in the total number of kilometers driven by the household can now be determined as:

$$
s_{i}=\frac{\frac{\partial P}{\partial p_{i}}}{\sum_{i \in O} \frac{\partial P}{\partial p_{j}}}, \quad i \in O .
$$

This equation shows that the distribution of total car kilometers over the individual cars is completely determined by the function $P$. According to the empirical studies discussed in the introduction, these shares are sensitive to the values of the variable costs of the cars owned by the household. Higher fuel prices should induce substitution towards the least expensive car, and a larger gap between the variable costs should have a similar effect on the use of all cars. The reason for this phenomenon must therefore be found in the specification of $P$. It may be observed, however, that the model as developed thus far excludes this phenomenon, since it assumes that all cars are identical. We will therefore next consider the extension of the model to cases in which cars can differ in fixed and variable costs.

\subsection{Car quality choice}

In reality there are many brands and makes of automobiles and, even though this paper will not concentrate on quality choice, the observation made at the end of the subsection 2.4 above clearly indicates the desirability of taking this heterogeneity into account in our model. In order to do so, we assume that cars are distinguished by quality $z$. Quality should be interpreted as a composite scalar measure of those car characteristics to which consumers attach a positive value, e.g. acceleration power, space, presence of airbags, air-conditioning. Variable and fixed costs are assumed to be increasing functions of quality. Although the number of available car brands and makes is finite in reality, their numbers seem large enough to justify working with a continuous approximation.

The treatment of quality as a scalar variable is less restrictive than it may appear to be at first sight. In many models of car type choice the utility associated with a particular brand or make is specified as a linear function of car quality characteristics and costs. The part of this function referring to the quality characteristics can then be interpreted as a scalar indicator of quality and if the number of car types is large, treating it as a continuous variable will not do much harm. Consider first a household with $N$ cars. Its utility function is given by (3) when all cars have the same quality. We now introduce the quality of the $N$ cars as separate variables, and take account 
of the dependence of costs on quality. For given qualities of the cars, the indirect utility function then becomes:

$v=v\left(y-\sum_{i=1}^{N} k\left(z_{1}\right), p\left(z_{1}\right), \ldots p\left(z_{N}\right), z_{1}, \ldots, z_{N}\right)$.

Maximization with respect to the quality of the cars gives first-order conditions:

$\frac{\partial v}{\partial\left(y-\sum_{i} k\left(z_{i}\right)\right)} \frac{d k\left(z_{i}\right)}{d z_{i}}+\frac{\partial v}{\partial p\left(z_{i}\right)} \frac{\partial p\left(z_{i}\right)}{\partial z_{i}}+\frac{\partial v}{\partial z_{i}}=0, \quad i=1, \ldots, N$.

We assume that these first-order conditions have a unique solution and that the second-order conditions are fulfilled. The optimal values for variable costs, fixed costs and quality will be denoted as $p_{i}^{o}, k_{i}^{o}$, and $z_{i}^{o}$, respectively, for $i=1, . ., n$. In general, they are functions of all parameters of the model, implying, for instance, that their values will change if the variable and fixed costs of car brands and makes change. Indirect utility can be written in terms of the optimal values of quality and fixed and variable costs as:

$v=v\left(y-\sum_{i=1}^{N} k_{i}^{o}, p_{1}^{o}, \ldots p_{N}^{o}, z_{1}^{o}, \ldots, z_{N}^{o}\right)$.

Now consider the case in which the household owns less than $N$ cars. We assume that $\partial u / \partial z_{i}=0$, whenever $x_{i}=0$. Virtual prices can still be derived for the non-owned cars, and since variable costs are an increasing function of quality, quality is determined by the value of the inverse of this function at the virtual price. This virtual quality also determines the virtual fixed costs of the non-owned cars.

For the owned cars, we can derive the optimal values of fixed and variable costs and quality in the same way as discussed above for the case in which $N$ cars are owned, assuming the conditions for a unique optimum are satisfied. Now let $k^{r}$ be the vector which has the optimal fixed cost $k_{i}^{o}$ as its $i$-th element when car $i$ is owned $\left(d_{i}=1\right)$ and the virtual fixed costs $k_{i}^{v}$ when car i is not owned $\left(d_{i}=0\right)$. The vectors $p^{r}$ and $z^{r}$ are defined analogously, and indirect utility is:

$v=v\left(y-\sum_{i=1}^{N} d_{i} k_{i}^{r}, p_{1}^{r}, \ldots p_{N}^{r}, z_{1}^{r}, \ldots, z_{N}^{r}\right)$.

When all $N$ cars are owned, $d$ contains only 1's, and (14) becomes identical to (13).

The utility $v_{n}$ of owning $\mathrm{n}$ cars is defined as:

$v_{n}=\max _{d \in D(n)}\left\{v\left(y-\sum_{i=1}^{N} d_{i} k_{i}^{r}, p_{1}^{r}, \ldots p_{N}^{r}, z_{1}^{r}, \ldots, z_{N}^{r}\right)\right\}, \quad n=0,1, \ldots, N$

and the optimum number of cars $n^{*}$ is:

$n^{*}=\arg \max \left\{v_{i}, i=1, \ldots N\right\}$.

\subsection{Further specification of the composite price function}

We now discuss the specification of $\mathrm{P}$ that will be used in the empirical work to be reported in Section 4. Although we now allow the variable and fixed costs of the cars owned by the household to be different, we will not deal explicitly with quality differences until the end of this subsection.

In our empirical work, we use the following specification of $P$ : 


$$
P=\frac{1}{\gamma} \ln \left(\frac{\sum_{i=1}^{N} w_{i} e^{p_{i}}}{\sum_{i=1}^{N} w_{i}}\right),
$$

where $\gamma<0$ and the $w_{i}>0$ for all $i$. The expression in brackets is a weighted average of the exponentiated variable costs that have been multiplied by $\gamma$. The weights $w_{i}$ may be functions of disposable income and other variables. They reflect the importance of a particular car for the household. If the variable costs of all cars in the household are equal, the number of kilometers driven by car $i$ is approximately proportional to $w_{i}{ }^{7}$

Note that (for positive variable costs) the expression in brackets will be positive, but smaller than 1. Its logarithm is therefore negative, but since it is divided by $\gamma$, the price indicator $P$ is always positive.

After taking the logarithm of the weighted sum and dividing through $\gamma$, we arrive at something that can be interpreted as some kind of average variable costs. Indeed, it is not difficult to verify that $P$ takes on a value between the minimum and the maximum of the variable costs of the various automobiles that could be used.

Even though $P$ can be interpreted as a kind of average price, (17) is not homogeneous of degree 0 in the variable costs $p_{i}$. Note, however, that this property is undesirable in the present context. The reason is that it would imply that the shares $s_{i}$, being the first derivatives of $P$, are homogeneous of degree 0 in these variable costs. A proportional change in all variable costs occurring, for instance, as a result of an increase in the fuel price would therefore leave these shares unchanged. As noticed in the introductory section, there is some empirical evidence that such a change would induce substitution towards the car with the lowest variable costs. In the context of the present model, it is therefore desirable that an identical percentage change in all variable costs does not lead to a change in $P$ by the same percentage.

The shares of automobile kilometers implied by (17) are:

$$
s_{i}=\frac{w_{i} e^{\imath p_{i}}}{\sum_{j=1}^{N} w_{j} e^{\eta_{j}}} \text {. }
$$

It is instructive to see what happens to the shares if the fuel price changes. It seems reasonable to assume that all variable costs are proportional to the fuel price, at least approximately. This means that all variable costs change by the same percentage if the fuel price changes. As a consequence, the relative decrease in $\exp (\gamma p)$ will be largest for the car with the highest variable costs and smallest for the car with the lowest variable costs. The share of the most expensive car in the household's car kilometers will therefore decrease, while that of the least expensive car will increase.

In a two-car household, a larger gap between the variable costs of the cars will imply a decrease in the use of the expensive car (in terms of variable costs) and an increase in that of the cheap car. When there are three or more cars present, higher variable costs for the most-expensive car means a larger gap with the costs of all other cars and a lower share of that car. Similarly, lower variable costs for the least-expensive cars means a larger gap with the costs of all other cars and a larger share of car kilometers for this least-expensive car.

\footnotetext{
${ }^{7}$ Compare equation (18) below.
} 
We may conclude that specification (17) is able to reproduce a household's typical responses to differences in variable car costs and changes in fuel prices, as reported in the literature discussed in the Introduction.

The effects of restrictions on the household's car ownership are readily analyzed. The virtual prices for the car or cars that are not owned are the prices that make the shares of these cars equal to 0 . All virtual prices are therefore infinitely positive, implying that $\exp \left(\gamma p^{v}\right)$ equals 0 . This means that the shares of the cars owned by the household are determined as if only the cars in a set $O$ are available to the household. Note, however, that there is an effect of rationing on $P$, because the positive weights $w_{i}$ of the cars not owned by the household remain present in the denominator of the ratio in (17).

Next, we note that (12) has as an additional attractive property, viz. that the sum of its partial derivatives with respect to the variable costs equals 1 :

$\sum_{i=1}^{N} \frac{\partial P}{\partial p_{i}}=1$.

As a result of this property, equation (9) which gives the total number of kilometers driven by the household, can be simplified to:

$$
X=-\frac{\frac{\partial v^{\prime}}{\partial P}}{\frac{\partial v^{\prime}}{\partial y^{\prime}}+\frac{\partial v^{\prime}}{\partial P} \frac{\partial P}{\partial y^{\prime}}} .
$$

The right-hand-side is Roy's identity for the total number of kilometers interpreted as a single good with price $P$. Equation (20) thus shows that the total demand for car kilometers can be determined as if $P$ were the price for car kilometers in general. If the partial derivative of $P$ with respect to disposable income equals 0 , this total number of kilometers is completely determined by the function $v^{\prime}$, and there is a clear distinction between trip generation (through the function $v^{\prime}$ ) and trip distribution (through the function $P$ ).

The previous discussion has ignored differences in car quality, but, before concluding this subsection, we have to consider its role in the further specification of the model. We have interpreted $P$ as a cost of car mobility, and higher car quality should be expected to lower this price. At first sight, it may seem attractive to introduce quality as one of the determining variables of the weights. However, this would bring us into conflict with the requirement that $\partial v / \partial z_{i}$, which is equal to $(\partial v / \partial P)\left(\partial P / \partial z_{i}\right)$, must be equal to 0 when the demand for kilometers for car $i$ is equal to 0 . The alternative possibility is that we reformulate $P$ in terms of qualityadjusted prices $r_{i}$ for automobile services:

$P=\frac{1}{\gamma} \ln \left(\frac{\sum_{i=1}^{N} w_{i} e^{m_{i}}}{\sum_{i=1}^{N} w_{i}}\right)$

with

$r_{i}=r\left(p_{i}, z_{i}\right)$, 
and we require that $\lim _{p_{i} \rightarrow \infty} r=\infty$. This formulation seems to be a reasonable one, and it covers, for instance, the repackaging model of Fisher and Shell (1971), which has $r_{i}=p_{i} / z_{i}$ as the price per unit of quality. ${ }^{8}$

\subsection{A generalization of de Jong's model}

The previous subsections have examined the way a household uses the cars it has available and the number of cars to be owned. The discussion thus far has been cast in general terms. It will now be made more concrete by choosing a particular specification of the (indirect) utility function. For this purpose, we adopt de Jong's (1990) specification of the indirect utility function: $v^{\prime}\left(y^{\prime}, P\right)=\frac{1}{1-\alpha}\left(y^{\prime}\right)^{1-\alpha}+\frac{1}{\beta} \exp (\delta-\beta P)$.

De Jong used the price of a single car in this function, but we have imputed the composite price $P$ specified according to (16) in order to be able to deal with cases in which the number of cars exceeds 1 .

If $P$ does not depend on disposable income, the total number of kilometers driven by the household is given by the following demand equation: $\ln (X)=\delta+\beta P+\alpha \ln \left(y^{\prime}\right)$.

A more complicated expression (that follows from (20) and is not discussed here) results if $P$ depends on disposable income.

If a household does not own all $N$ cars, demand for kilometers of the non-owned cars should be equal to 0 . The same must be true for the shares of the kilometers driven by these cars in total demand for kilometers, and it is easily seen from (17) that the virtual prices at which this happens are (positive) infinite. ${ }^{9}$ Since $\gamma$ is negative, this means that these variable costs disappear from the numerator in (16). However, their weights remain present in the denominator, and this implies that $P$ will be higher when a car is removed. In other words: the effect of rationing associated with owning a limited number of cars is a higher composite price $P$ of car kilometers.

If a household owns one car, the value of $P$ is:

$P=p_{i}+\ln \left(w_{i} / \sum w_{j}\right) / \gamma$.

Limited possibilities for substitution between the use of different cars imply a value of $\gamma$ that is close to 0 , leading to a value of $P$ under rationing that is much higher than $p_{i}$. In other words, limited possibilities for substitution imply large costs of rationing.

If the formula for $P$ in the case where just one car is owned is substituted into (22), the second term on the right-hand side can be absorbed into $\delta$. The resulting indirect utility function is the one used by de Jong (1990) for single-car-owning households. ${ }^{10}$

\footnotetext{
${ }^{8}$ It must be noted, however, that in the model of Fisher and Shell, the varieties (which correspond in the present context to the various cars owned by the household) are perfect substitutes, which is not useful for the purposes of the present paper.

9 The chosen specification of $P$ implies that cars will be used whenever they are owned.

${ }^{10}$ De Jong (1991) has proposed a different extension of the (1990) model to situations in which households can own two cars. It is based on a utility function $v^{\prime}=\left(y^{\prime}\right)^{1-\alpha}+\exp \left(\delta_{1}+\beta_{1} p_{1}\right) / \beta_{1}+\exp \left(\delta_{2}+\beta_{2} p_{2}\right) / \beta_{2}$. This leads to demand equations for the kilometers driven by both cars in which the variable costs of the other car does not appear. There are therefore no substitution effects present in this model.
} 


\section{The data}

In this section we introduce the data set used in this paper, discuss some aspects of these data that are of special interest and present reduced form equations for variable and fixed costs of the cars.

\subsection{Description of the data}

The data set used in this paper originates from three different data sources:

1 the 'car panel' conducted by the Dutch Consumer's Association (Consumentenbond)

2 a household survey that supplemented the car panel questionnaire in the period 1992-1994

3 technical car attributes provided by the Dutch Motorists' Association (ANWB).

The 'car panel' constitutes the basis of the data set. It contains information about 6000 passenger cars. Participants are recruited among the members of the association with the primary purpose of generating information about the costs of the most popular car types. ${ }^{11}$ Twice a year, the panel car owners have to fill in a form with questions about repair and maintenance costs, number of kilometers recorded, and attributes of the panel car. Mileage is also recorded then.

The information gathered for the car panel was supplemented in the period 1992-1994 by a household survey, conducted as part of a publicity campaign that started towards the end of 1992, and which was initiated by the Dutch Agency for Energy and Environment (Novem). The campaign was aimed at achieving a more fuel-efficient car-use and acquisition behavior among passenger car owners. In order to monitor the effects of the campaign, data were collected from the participants of the car cost panel of the Dutch Consumer's Association in October/November of the consecutive years 1992, 1993 and 1994. In addition to the information gathered for the panel car cost, respondents were also questioned about the presence of a second and third car and the number of kilometers driven with these cars. This additional information was crucial for the purposes of the present paper.

Table 1 Descriptive statistics

\begin{tabular}{|c|c|c|c|c|c|}
\hline & Variable & Mean & Std Dev & Min & Max \\
\hline \multicolumn{6}{|l|}{ Households } \\
\hline & Income $(x 1000)$ & 27.39 & 9.31 & 5 & 45 \\
\hline & Number of workers & 1.11 & 0.86 & 0 & 5 \\
\hline & Number of persons & 2.69 & 1.15 & 1 & 7 \\
\hline & Number of licensed drivers & 1.78 & 0.58 & 1 & 5 \\
\hline & Number of company cars & 0.019 & 0.14 & 0 & 2 \\
\hline & Second car present & 0.11 & 0.32 & 0 & 1 \\
\hline \multicolumn{6}{|l|}{ Cars } \\
\hline & Monthly km & 1555 & 842 & 2 & 7337 \\
\hline & Variable costs & 0.18 & 0.03 & 0.08 & 0.27 \\
\hline & Fixed costs & 3551 & 1278 & 1645 & 8251 \\
\hline & Main driver is male & 0.81 & & & \\
\hline
\end{tabular}

Number of observations: 3180 households. Households with second car: 357 . Number of observed cars: 3537 .

${ }^{11}$ There are 214 car types for which information is collected. Cars can be included in the panel when: a) the odometer must not exceed 150,000 kilometres, b) the car is at most 10 years old, and c) the car covers at least 5,000 but at most 40,000 kilometers a year. 
The technical information, that constitutes the third part of our data concerns (i) car attributes; (ii) fuel consumption; (iii) fixed and variable depreciation rates; (iv) market prices of new and second-hand versions of each of the 214 make/model combinations; and (v) the time period in which the version was available for purchase on the market. The figures referring to average and variable costs that are used in this paper originate from this data source.

The data from these three sources were amalgamated into six waves of panel data. In the present paper we use the 4th wave, which refers to the second half of 1993. Some descriptive statistics are presented in Table 1.

The average annual mileage of the cars in our sample is very close to that of the Dutch car fleet. The households in our sample have slightly more members than the Dutch households, company cars appear to be underrepresented and the average age of the car owners in our sample is slightly higher than that in the total Dutch population.

\subsection{Discussion of some aspects of the data}

A second car is, of course, especially useful if there is more than one person with a driver's license in the household. ${ }^{12}$ This suggests that one should estimate a model for multiple car ownership only on data referring to households with at least two licensed driver. However, estimation of separate probit models for the choice of either one or two cars for households with one and two or more licensed drivers resulted in coefficients that are not significantly different. ${ }^{13}$ A possible explanation of this result is that the number of driver's licenses is adjusted to the need for a second car. We have therefore estimated the model on data referring to all households. A second issue that needs discussion is the use of a company car. Such cars have become much more popular in the Netherlands since fiscal legislation was changed in the early 1990s. Company cars are usually, but certainly not exclusively, offered to workers who have to drive a lot for business purposes. Because of its relation with travel for business purposes, one could regard the company car as a more or less exogenous addition to the household's vehicle stock. However, there are several reasons for questioning the appropriateness of such an approach. In many firms a large number of employees may opt for a company car, regardless of the need to drive for business purposes. Choosing this option means that their salary is lower. Moreover, workers with a company car have to add part of its value to their taxable income, which means that they have to pay some fixed costs. The kilometers they drive for business purposes and also home-work trips are usually paid by their employers, but many workers have to pay variable (fuel) costs themselves if the number of private kilometers exceeds a predetermined threshold. This means that, in many cases, the relevant marginal costs are equal to the fuel cost. Even though the choice of car brand and make for company cars is not completely free, most workers can choose from a broad range, which is sometimes related to the type of job.

The previous discussion suggests that workers with a company car are not too different from workers who have bought their car themselves and that there is no need to use a completely different model specification for households with and households without such a car. In the model estimated in the next section, we will use a dummy for company cars as an indicator of the demand for kilometers.

\footnotetext{
${ }^{12}$ In the sample, there are 904 households with one driver's license, 9 of them have two cars.

${ }^{13}$ Estimation results are available upon request.
} 


\subsection{Determinants of car costs}

We indicated in the previous section that the present paper will not focus on quality choice, even though we cannot ignore that issue, since substitution between the use of the two cars is related to differences in costs that are related to quality differences. In Section 2.5 we outlined a model that incorporates quality choice. Higher quality could be realized at the expense of higher fixed and variable costs. Presumably, the trade-off between benefits and costs depends presumably on household characteristics, and the reduced form equations of such a submodel would therefore relate fixed and variable costs to household characteristics. In this subsection, we discuss estimation results of such reduced-form equations. We start with variable car costs.

Table 1 above shows that the variation in variable costs is limited: the figures are all in the interval $[0.08,0.27]$. Casual evidence suggests, however, that it may nevertheless be interesting to investigate the determinants of the differences. Estimation results of a linear regression are shown in Table 2. They confirm the common-sense opinion that males drive cars with higher variable costs, even though the difference is not large. When household income is higher, variable costs tend to go up. However, given income (and the values of the other explanatory variables) variable costs tend to be slightly lower if the number of workers is larger. The presence of more licensed drivers is associated with higher variable costs, but the presence of more cars is associated with lower variable costs. There is no difference in this respect between cars with a male or a female main driver. Higher fixed costs are often associated with heavier cars, implying more fuel use per kilometer and thus higher variable costs, a relationship that is confirmed by the significant positive coefficient for fixed costs.

\section{Table 2 Variable costs per kilometer and household characteristics}

\begin{tabular}{ll}
\hline Variable & Estimate (st. error) \\
\hline Constant & $\mathbf{0 . 1 6 9}(0.003)$ \\
Male & $\mathbf{0 . 0 0 6 6 1}(0.0019)$ \\
Income (x1000) & $\mathbf{0 . 2 7 7}(0.058)$ \\
Number of workers & $\mathbf{- 0 . 0 0 5 0 8}(0.0007)$ \\
Company car & $-0.00255(0.0031)$ \\
Household size & $-0.000599(0.00052)$ \\
Number of licensed drivers & $\mathbf{0 . 0 0 3 2 8}(0.0010)$ \\
Second car present & $\mathbf{- 0 . 0 0 7 7 8}(0.0025)$ \\
Male*second car present & $0.00224(0.029)$ \\
Fixed cost (x1000) & $\mathbf{0 . 0 0 1 5 6}(0.004)$ \\
& \\
$R^{2}$ & 0.052 \\
$n$ & 3537 \\
\hline
\end{tabular}

Standard errors are reported in parentheses. Coefficients in bold print are significant at $\mathrm{p}=.05$

Table 3 reports estimation results that refer to the logarithm of fixed car costs. Here we find a substantial effect of the gender of the main driver. Males use cars that have, on average, more than $10 \%$ higher fixed costs than females. A higher income is associated with higher fixed car costs, and if the number of workers increases these costs tend to be even higher. Company cars tend to have higher fixed costs. The presence of a second car is associated with lower fixed costs, and again we find no difference in this respect between cars whose main driver is male and those whose main driver is female. 
As mentioned above, we will use these estimation results to predict the fixed and variable costs of the single car or the two cars used by the households in our sample, in order to estimate the structural model. This means that we will interpret the predictions of the fixed and variable costs from these equations as the optimal values implied by a model of quality choice. The estimated equations give reasonable values for the fixed and variable costs. For instance, the average predicted fixed costs for a household with one car is 4052 Dutch guilders, whereas the average fixed costs of two-car households are 3359 Dutch guilders for the male driver and 3006 Dutch guilders for the female driver. Average predicted variable costs are in all three cases between 0.17 and 0.19 Dutch guilders per kilometer.

Table 3 Fixed cost

\begin{tabular}{ll}
\hline Variable & Estimate (st. error) \\
\hline Constant & $\mathbf{7 . 5 9 4}(0.06)$ \\
Male & $\mathbf{0 . 1 3 0}(0.02)$ \\
Ln(income) & $\mathbf{0 . 1 2 1}(0.02$ \\
Number of workers & $\mathbf{0 . 0 2 1}(0.009)$ \\
Company car & $\mathbf{0 . 1 3 6}(0.04)$ \\
Household size & $-0.00827(0.006)$ \\
Number of licensed drivers & $0.0185(0.013)$ \\
Second car present & $\mathbf{- 0 . 0 7 7 0}(0.030)$ \\
Male*second car present & $0.0155(0.035)$ \\
& \\
$R^{2}$ & 0.044 \\
$n$ & 3537 \\
\hline
\end{tabular}

Dependent variable: $\log ($ Fixed Cost). Standard errors are reported in parentheses. Coefficients in bold print are significant at $\mathrm{p}=.05$.

\section{Estimation of the structural model}

\subsection{Introduction}

In this section, we discuss the estimation of the structural model derived in Section 2.7. Given the data discussed in the previous section, the model is focused on the choice to own one or two cars. Accordingly, it takes the maximum number of cars per household to be equal to two and the minimum to be one ${ }^{14}$

We will not make a distinction between first and second cars, since it would force us to introduce an element of arbitrariness into the model. There is no intrinsic reason why one car should be labeled "primary" and the other "secondary." We have therefore treated the cars symmetrically. There is, of course, good reason to suppose that car use will be dependent on the characteristics of the main driver. For this reason we have related differences in car use to differences in driver characteristics. More specifically, we have distinguished between cars with male and those with female main drivers.

\subsection{The likelihood function}

\footnotetext{
${ }^{14}$ We deleted 22 households with more than two cars.
} 
We use the estimated equations for fixed and variable costs to predict the values of these variables for all households in our sample when they own a single car and two cars. The values for the fixed costs will be denoted as $k_{1}$ when a single car is owned, and as $k_{2,1}$ and $k_{2,2}$ when two cars are owned. The composite prices for one and two cars are computed on the basis of the predicted variable costs and will be referred to as $P_{1}$ and $P_{2}$, respectively. In order to get some idea of the determinants of these variables, we have separately estimated share equations that follow from (21). ${ }^{15}$ An important result was that the coefficient for household income was never significant, which implies that (23) can be used as the demand equation for the total number of kilometers driven by the household. Another feature that should be mentioned is the treatment of quality. Since quality itself is unobserved, we included fixed car costs as an indicator, but we were unable to find a significant coefficient in the various specification we tried. Apart from the variable cost, only the gender of the main driver appear to have a significant effect on the distribution of the total number of kilometers over the two cars. On the basis of these estimation results, we have chosen parsimonious specification of the composite price that includes only these two variables:

$$
P_{1}=\frac{1}{\gamma} \ln \left(\frac{e^{\theta_{1} I(\text { male })+p_{1}}}{1+e^{\theta_{1}}}\right)=p_{1}+\frac{1}{\gamma} \ln \left(\frac{e^{\theta_{1} I(\text { male })}}{1+e^{\theta_{1}}}\right)
$$

and

$$
P_{2}=\frac{1}{\gamma} \ln \left(\frac{e^{\theta_{1}+2 p_{2,1}}+e^{\imath p_{2,2}}}{1+e^{\theta_{1}}}\right),
$$

where $I($.$) is an indicator variable that is equal to 1$ if the male driver is male and equal to 0 otherwise. For two-car households, we have adopted the convention that car 1 is the one used by the male driver.

The specification of the indirect utility function of de Jong (1990), was given in (22). The utility of owning a single car is:

$u^{1}=\frac{1}{1-\alpha}\left(y-k_{1}\right)^{1-\alpha}+\frac{1}{\beta} \exp \left(\delta-\beta P_{1}\right)$

and that of owning two cars:

$\left.u^{2}=\frac{1}{1-\alpha}\left(y-k_{2,1}-k_{2,2}\right)^{1-\alpha}+\frac{1}{\beta} \exp \left(\delta-\beta P_{2}\right)\right)$.

Owning a single car implies lower fixed car costs, but a higher composite price for car mobility. The costs of owning two cars thus appear in the first term on the right-hand side of the above equations, while the benefits emerge in the second term. The magnitude of the benefits is dependent on the value of the random variable $\delta$, which is assumed to be a drawing from a normal distribution with mean $\mu_{\delta}$ and standard deviation $\sigma_{\delta}$. It can therefore be written as:

$$
\delta=\mu_{\delta}+\sigma_{\delta} \varepsilon \text {, }
$$

with $\varepsilon$ a drawing from the standard normal distribution. If $\varepsilon$ is sufficiently large, the utility of owning two cars will exceed that of owning a single car. The critical value $\varepsilon^{*}$ can be determined as:

$$
\varepsilon^{*}=\frac{1}{\sigma_{\delta}} \ln \left(\frac{\beta}{1-\alpha} \frac{(y-k)^{1-\alpha}-(y-2 k)^{1-\alpha}}{\exp \left(\mu_{\delta}\right)\left(\exp \left(-\beta P_{2}\right)-\exp \left(-\beta P_{1}\right)\right)}\right) \text {. }
$$

\footnotetext{
${ }^{15}$ Estimation results are available upon request.
} 
The probability of owning two cars is equal to the probability that $\varepsilon$ exceeds its critical value, and the probability of owning one car is its complement. The parameter $\mu_{\varepsilon}$ is further specified as a linear function of household characteristics $h$ :

$\mu_{\delta}=\delta_{0}+\sum_{k} \delta_{k} h_{k}$.

The outcome of the choice between owning two cars or one is now formulated as a non-linear probit model.

The number of kilometers driven by the household is determined conditional on the chosen number of cars. The random variable $\delta$ appears in the demand equation (23), and we must account for the truncation caused by the car ownership decision. Following de Jong (1990), we add a normal distributed random term $\eta$ (with expectation 0 and variance $\sigma_{\eta}$ ) to this equation in order to take into account measurement and optimization errors, and estimate:

$$
\begin{aligned}
\ln (X) & =\delta_{0}+\sum_{k} \delta_{k} h_{k}+E(\varepsilon \mid m \text { cars })+\beta P_{m}+\alpha \ln \left(y^{\prime}\right)+\eta \\
& =A(m)+\eta
\end{aligned}
$$

for $\mathrm{m}=1,2$, the number of cars owned by the household. When one car is owned, $\varepsilon \leq \varepsilon^{*}$, and when two cars are owned $\varepsilon>\varepsilon^{*}$. The conditional expectation for $\varepsilon$ in (31) can be determined with standard formulas given, for instance, in Verbeek (2000).

We are now able to formulate the likelihood function. The likelihood $l_{i}$ of an observation $i$ is the product of the probability that $\varepsilon$ is in the relevant range multiplied by the value of the normal density for the $\eta$ that follows from setting (30) equal to the observed total number of kilometers. Using a suffix $i$ to distinguish observations, this gives:

$\left.\left.l_{i}=I\left(m_{i}=1\right)\right) \operatorname{Pr}\left(\varepsilon \leq \varepsilon_{i} *\right) \phi\left(\frac{A_{i}(1)-\ln \left(X_{i}\right)}{\sigma_{\eta}}\right)+I\left(m_{i}=2\right)\right) \operatorname{Pr}\left(\varepsilon>\varepsilon_{i} *\right) \phi\left(\frac{A_{i}(2)-\ln \left(X_{i}\right)}{\sigma_{\eta}}\right)$,

where $I($.$) is the indicator variable, and \sigma_{\eta}$ is the standard deviation of $\eta$.

In order to estimate the parameters of $P$ simultaneously with the other parameters, we substitute the expression given in (25) into (29) and (31) and the resulting expressions in (32).

It may be observed that the likelihood formulated in (32) does not use the information we have on the distribution of the total number of kilometers of households owning two cars over both cars.

In order to use this information as well, note that the demand kilometers of car $k=1,2$, to be referred to as $X_{k}$, is determined as $X_{k} s_{k}$, with $s_{k}$ the share of this car in household kilometers. Equation (18) shows how this share is related to the composite price $P$. The shares implied by the parsimonious formulation of $P$ are:

$s_{2,1}=\frac{e^{\theta_{1}+2 p_{2,1}}}{e^{\theta_{1}+p p_{2,1}}+e^{2 p_{2,2}}}$

and

$s_{2,2}=\frac{e^{\not p_{2,2}}}{e^{\theta+2 p_{2,1}}+e^{\imath p_{2,2}}}$.

The demand for kilometers that is predicted by the model equals total demand for kilometers by the household times the share of the particular car. We assumed that the error in the demand equation for each car (on one- and two-car-owning households) is a normal distributed random variable whose standard deviation should be estimated. We allow the errors in the demand for kilometers of two cars in the same household to be correlated. 
Formally, the demand equations to be estimated for the households owning two cars are:

$$
\begin{aligned}
\ln \left(X_{k}\right) & =\delta_{0}+\sum_{k} \delta_{k} x_{k}+E(\varepsilon \mid 2 \text { cars })+\beta P_{m}+\alpha \ln \left(y^{\prime}\right)+\ln \left(s_{k}\right)+\eta_{k} \\
& =B_{k}+\eta_{k},
\end{aligned}
$$

where $s_{k}$ denotes the kilometer share of the $k$-th car as given in (33) for $N=2$. The likelihood function for observation $i$ now becomes:

$$
\begin{gathered}
l_{i}=I\left(m_{i}=1\right) \operatorname{Pr}\left(\varepsilon \leq \varepsilon_{i} *\right) \phi\left(\frac{A_{i}(1)-\ln \left(X_{1 i}\right)}{\sigma_{\eta}}\right)+ \\
I\left(m_{i}=1\right) \operatorname{Pr}\left(\varepsilon>\varepsilon_{i}^{*}\right) f\left(\frac{B_{1 i}-\ln \left(X_{1 i}\right)}{\sigma_{\eta}}, \frac{B_{2 i}-\ln \left(X_{2 i}\right)}{\sigma_{\eta}}, \rho\right),
\end{gathered}
$$

where $f$ denotes the density of the bivariate normal, and $\rho$ the correlation between the two variates.

Table 4 Estimation results for the ownership/use model

\begin{tabular}{llcl}
\hline & & Total demand & $\begin{array}{l}\text { Demand for ind. } \\
\text { Cars }\end{array}$ \\
\hline Symbol & Variable & Estimate (st. err.) & Estimate (st err.) \\
\hline$\alpha$ & Income & $\mathbf{0 . 1 3 5}(0.0093)$ & $\mathbf{0 . 1 3 2}(0.0088)$ \\
$\beta$ & Price & $\mathbf{- 0 . 5 0 8}(0.037)$ & $\mathbf{- 0 . 3 6 9}(0.049)$ \\
$\delta_{0}$ & Constant & $\mathbf{6 . 4 0}(0.12)$ & $\mathbf{6 . 2 4}(0.12)$ \\
$\delta_{1}$ & Number of workers & $\mathbf{0 . 0 2 8 5}(0.004)$ & $\mathbf{0 . 0 3 0 3}(0.004)$ \\
$\delta_{2}$ & Company car & $\mathbf{0 . 1 8 6}(0.014)$ & $\mathbf{0 . 1 8 6}(0.014)$ \\
$\delta_{3}$ & Household size & $-0.00264(0.0028)$ & $-0.00214(0.0028)$ \\
$\delta_{4}$ & Number of licensed drivers & $\mathbf{0 . 4 8 2}(0.077)$ & $\mathbf{0 . 0 5 2 2}(0.0077)$ \\
$\sigma_{\eta}$ & S.e. demand equation & $\mathbf{0 . 5 1 7}(0.0025)$ & $\mathbf{0 . 4 6 1}(0.0024)$ \\
$\sigma_{\delta}$ & S.e. idiosyncratic demand & $\mathbf{0 . 0 2 3 6}(0.0050)$ & $\mathbf{0 . 0 2 4 0}(0.0050)$ \\
$\theta_{1}$ & Male & $\mathbf{0 . 2 6 5}(0.044)$ & $\mathbf{0 . 2 7 9}(0.034)$ \\
$\gamma$ & Difference in variable cost & $\mathbf{- 0 . 4 9 9}(0.010)$ & $\mathbf{- 0 . 4 6 6}(0.012)$ \\
$\rho$ & Correlation between errors & & $-0.00786(0.025)$ \\
& in demand equations & & \\
& & $-2,509.04$ & $-2,607.95$ \\
& Loglikelihood & 3165 & 3165 \\
\hline
\end{tabular}

Note. Households with an income less than two times the (average) fixed costs of car ownership were removed from the sample.

\subsection{Estimation results}

The model that has been formulated in the Section 4.4 above is highly non-linear in the parameters, and we started estimation with a two-step procedure, in which the results of separate estimation of the parameters of the share equation (33) were used to compute the composite price function and these values were imputed in the likelihood function (32). The other parameters were then estimated. In this way, we obtained starting values for all parameters of the model. We then used (32) to obtain simultaneous estimates of all the parameters. Likelihood function (35) 
was then estimated with the results of estimating (34) as starting values. Estimation results are given in Table 4, where the column labeled 'total demand' refers to likelihood function (32), and the one labeled 'demand for individual cars' to (35).

The parameters $\alpha$ and $\beta$ have the expected signs and are significant. The former can be interpreted as the income elasticity of the total demand for kilometers, and the price elasticity is equal to $\beta P$. The income elasticities implied by the two models are almost equal, but the model based on individual car kilometers implies a smaller (absolute) value for the price elasticity. The number of workers has a positive effect on the demand for car kilometers and on the probability of owning two cars. There is a similar effect of having a company car. The coefficient for household size is insignificant. There is large and highly significant effect of the number of licensed drivers in the model based on total kilometers driven by the household. The coefficient that measures this effect becomes much smaller if we estimate the model on individual car kilometers, but it remains highly significant. The (absolute) value of the coefficient $\gamma$ is small, but highly significant.

We have also experimented with more explanatory variables for the composite price function and the share equations, but we were unable to find more significant coefficients. ${ }^{16}$

We have also tried a specification in which we allowed the price coefficient to be different for company cars, but we did not find a statistically significant difference.

\section{Discussion of the results and an application}

In this section, we discuss the interpretation of the estimation results and provide an application to a change in transport policy, which has repeatedly been considered in the Netherlands, viz. a partial shift of the burden of taxation from fixed to variable car costs. Throughout the section, we focus on a model estimated on individual car kilometers, which is regarded as the preferred model specification.

\subsection{Estimation results: elasticities and substitution possibilities}

The parameter $\alpha$ is equal to the income elasticity of the demand for kilometers, conditional on the number of cars. Its value, .132, seems reasonable. The equivalent price elasticity is equal to $\beta P$, approximately -.07 . The absolute values of both elasticities are at the lower end of the range found in other studies ${ }^{17}$ (see, among others, Graham and Glaister, 2002, and Espey, 1998). A central feature of our model is the substitutability between the kilometers driven by the two cars. This substitutability is summarized by the coefficient $\gamma$, which is found to be highly significant in all variants of the model. The earlier literature referred to in the introduction suggests the significance of this substitution effect, and the structural model thus confirms this. Households tend to use the car with the lower variable costs more often when the difference between the variable costs of the two cars is larger.

In order to see what this implies for the effect of changes in the fuel price, the following equation is helpful:

$\gamma=\frac{d \ln \left(s_{1} / s_{2}\right)}{d\left(p_{1}-p_{2}\right)}$.

\footnotetext{
${ }^{16}$ This confirmed the results of the preliminary analysis in which the share equations were estimated separately.

${ }^{17}$ They are lower than those implied by the estimation results of the first variant, which were already on the low side. De Jong (1991) found a high absolute value for the price elasticity. Note, however, that our results do not include the possible effect of changes in driving style or in the choice of automobile quality that occur in reaction to changes in fuel prices.
} 
The coefficient $\gamma$ equals the change in the logarithm of the ratio of the kilometer shares induced by a change in the difference between the variable costs of the two cars. Further elaboration of this equation gives:

$d s_{1}=\gamma s_{1}\left(1-s_{1}\right) d\left(p_{1}-p_{2}\right)$.

Note that $s_{1}\left(1-s_{1}\right)$ is, at most, equal to .25 . Given the estimated value of $\gamma$, this implies that the change in the share of car 1 is, at most, $12.5 \%$ of the change in the gap between the two variable costs. This gap itself equals, on average, $.04 .{ }^{18}$ Since changes in this gap will usually be a (small) fraction of its value, it is clear that substitution between the two cars as a consequence of changes in variable costs will in general be small. For instance, if fuel prices are multiplied by $k$, the implied change in the variable cost gap equals $(k-1)\left(p_{1}-p_{2}\right)$, and a price increase of, for instance, $10 \%$ implies that $k$ equals 1.1 . It must therefore be concluded that, according to our estimation results, the substitution between the kilometers driven by cars induced by changes in fuel prices is limited. ${ }^{19}$

The possibility to substitute the use of one car for that of the other also plays an important role in the costs associated with having just one car. The price $P$ of car of a single-car household is: $P=p_{1}+\ln \left(w_{1} /\left(w_{1}+w_{2}\right)\right) / \gamma$.

If the variable costs of all cars are equal, the second term on the right-hand side equals the additional cost of car mobility caused by the absence of a second car. Since the variation in variable costs among cars is limited, the value of this term provides a good approximation. The typical single-car household has a male main driver, and the value of $\ln \left(w_{1} /\left(w_{1}+w_{2}\right)\right) / \gamma$ is then equal to 1.21. This additional cost is therefore a multiple of the variable costs, which are, on average, equal to .18. Our model estimates therefore suggest that the costs implied by the restriction of having a single car are substantial. These high costs are the mirror image of the limited possibilities for substitution between the kilometers driven by the two cars that were discussed above.

Effects of price and income changes on car ownership can be computed by simulating a 1\% increase in variable car costs and income on the share of households in our sample which have two cars. The quasi elasticities are -.057 and .147 , respectively, meaning that a $1 \%$ increase in the variable car costs implies a decrease in the share of households owning two cars of .057, whereas the effect of a $1 \%$ increase in income is greater in magnitude, and of opposite sign. The price elasticities of the demand for kilometers of one- and two-car households are -.065 and -.062, respectively, and the total effect of a 1\% change in variable costs on car kilometers equals -.098. The difference is caused by the fact that some second cars are abandoned in reaction to the price increase. Similarly, the total effect of a 1\% change in income on the total number of car kilometers of one and two-car households is .16\% and .18\%, respectively. However, the total effect of an increase in income is much larger due to the high income elasticity of demand for second car ownership: it equals 2.4 .

\subsection{Application: shifting the burden of taxes from fixed to variable costs}

\footnotetext{
18 The gap is computed as the average absolute value of the difference between the variable costs of the two cars for two-car households.

${ }^{19}$ However, it may be noted that our discussion assumes that both cars use the same fuel type. Larger effects may be realized if one car uses gasoline and the other diesel, and the price of one fuel type changes, while that of the other remains constant.
} 
We will now use the estimated model to study the effect of shifting the burden of taxation from fixed car costs to variable car costs. In general, one expects a decreasing effect of such a 'variabilization' of car taxes on the number of car kilometers, since the marginal costs of driving increases. However, the lower fixed costs will increase car ownership, and, taking this into account, not only for owning a single car but also for two cars, may change the picture. In order to investigate this question, we considered a decrease in fixed car costs of 120 Dutch guilders per year, financed by an increase in variable car costs of 0.70 Dutch guilder per kilometer. If car ownership and the number of kilometers driven with the cars remain unchanged, these changes would have no effect on the government budget. The decrease in fixed car costs can be realized by, for instance, a decrease in the car tax. We consider two variants: the decrease in the tax may be the same for all cars, or it may be proportional to the fixed costs themselves (although, on average, still equal to 120 guilders per year). The actual car tax in the Netherlands depends on the weight of the car. For the change in variable costs, we also consider two possibilities: it is either equal for all cars, or proportional to the variable costs themselves. The first may be interpreted as a kilometer charge, the second as an increase in the car fuel tax. Table 12 gives the effects of the various combinations. The first panel of the table gives the effects of the change in fixed costs only, the second panel that of the change in the variable costs only, and the third the combined effect of the two measures.

Table 5 Effects of shifting taxes from fixed to variable costs

\begin{tabular}{|c|c|c|c|c|}
\hline & $\begin{array}{l}\text { Equal decrease in } \\
\text { fixed costs, } \\
\text { Equal increase in } \\
\text { variable costs }\end{array}$ & $\begin{array}{l}\text { Equal decrease in } \\
\text { fixed costs, } \\
\text { prop. increase in } \\
\text { variable costs }\end{array}$ & $\begin{array}{l}\text { Prop. Decrease in } \\
\text { fixed costs, } \\
\text { Equal increase in } \\
\text { variable costs }\end{array}$ & $\begin{array}{l}\text { Prop. decrease in } \\
\text { fixed costs, } \\
\text { prop. increase in } \\
\text { variable costs }\end{array}$ \\
\hline \multicolumn{5}{|c|}{ Effect of change in fixed costs } \\
\hline $\begin{array}{l}\text { Change in share } \\
\text { of two-car } \\
\text { household }\end{array}$ & 0.0465 & 0.0465 & 0.026 & 0.026 \\
\hline $\begin{array}{l}\% \text { change in } \\
\text { kilometers driven }\end{array}$ & $3.12 \%$ & $3.12 \%$ & $1.82 \%$ & $1.82 \%$ \\
\hline \multicolumn{5}{|c|}{ Effect of change in variable costs } \\
\hline $\begin{array}{l}\text { Change in share } \\
\text { of two-car } \\
\text { household }\end{array}$ & -0.0024 & -0.0021 & -0.0024 & -0.0021 \\
\hline $\begin{array}{l}\% \text { change in } \\
\text { kilometers driven }\end{array}$ & $-0.42 \%$ & $-0.38 \%$ & $-0.42 \%$ & $-0.38 \%$ \\
\hline Effect of the comb & ned changes & & & \\
\hline $\begin{array}{l}\text { Change in share } \\
\text { of two-car } \\
\text { household }\end{array}$ & 0.0441 & 0.0443 & 0.0239 & 0.0241 \\
\hline $\begin{array}{l}\% \text { change in } \\
\text { kilometers driven }\end{array}$ & $2.71 \%$ & $2.74 \%$ & $1.40 \%$ & $1.44 \%$ \\
\hline
\end{tabular}

The most salient feature of the figures in the table is that our model implies relatively large effects of the change in fixed costs. In order to interpret this finding, it may be noted that the small estimated value of $\alpha$ implies that indirect utility is almost linear in income minus fixed 
automobile costs (see eqs. 28 and 29). A change in the difference between the fixed costs of having one or two cars thus implies a change in the difference between the associated indirect utilities of approximately the same size. Even though the effect of this change on the probability of having two cars runs via a logarithmic transformation (see eq. 30), it is large in comparison with the effect of the change in the variable costs considered here. ${ }^{20}$ If the change in fixed costs is equal for all cars, the effect on the difference in disposable income is always equal to 120 Dutch guilders per year. If the change is made proportional to fixed costs, the effect is much smaller. The reason is that, if a household owns a single car, it is usually a relatively expensive one in terms of fixed costs in comparison with the two cars that would otherwise have been owned. Indeed, the change in the difference between the disposable incomes when owning one or two cars is, on average, equal to 67 Dutch guilders under a proportional change in fixed costs, i.e. almost half of its value under a fixed change. It follows that a proportional change in fixed costs also has a much smaller impact on ownership of two cars, as is shown in the last two columns of Table 9.

The increase in variable costs has a much smaller impact. The reason is, of course, that the price elasticity is small. The impact on the prices $P_{1}$ and $P_{2}$ is therefore also limited. As a consequence, the effects on car ownership and on the number of kilometers driven are modest. Hence, the effect of the change in fixed costs dominates that of the simultaneous introduction of the changes in fixed and variable costs. In all four variants considered, the net result of the shift in taxes from fixed to variable costs is an increase in the number of kilometers driven. It should, therefore, be concluded that the effects of shifting the burden of car taxes from fixed to variable costs depend crucially on their influence on multiple-car ownership.

Note also that the model estimated in this paper concerns only the choice between one or two cars. Additional effects on car ownership may be caused by households who have not owned a car thus far but decide to buy one because of the tax policy measure, and by multiple car-owning households deciding to increase their number of cars even further. Note also that because we used imputed values of fixed and variable costs, we were unable to compute the possible effects of the policy measure on these variables through the choice of car brands and makes (quality).

\section{Conclusion}

In this paper, we have considered the demand for second cars on the basis of a general model for multiple car ownership and use. In the model we have estimated, there is imperfect substitutability between the use of the two cars potentially owned by a household. As a consequence, owning one car implies a loss in utility that may outweigh the lower fixed costs. Total demand for car kilometers is determined by a composite price for car kilometers that lies between the variable costs of the two cars. If one car is owned, this composite price is always higher than when two cars are owned. The lower composite price that is the result of owning two cars reflects the benefits of the second car to the household. These benefits are larger when substitution between the kilometers of the two cars is more difficult. The costs of owning two cars are higher fixed costs, which implies that less money is available for consumption goods. The preferred version of our model indicates that substitution between the kilometers driven by the two cars is relatively difficult, and the price sensitivity of total demand for car kilometers is relatively low. The latter result is in line with other studies of the demand for car kilometers, even though the (absolute value of the) price elasticity estimated here is on the low side of the range reported in the literature. The model was estimated on the basis of imputed cost figures that are

\footnotetext{
${ }^{20}$ It may also be noted that we haven't scaled disposable income when estimating the model.
} 
based on reduced-form regressions for fixed and variable car costs. Ideally, one would derive such equations from an explicit model of car quality choice. In such a model, a higher quality implies higher fixed and/or variable costs. Our car-cost regressions were interpreted as the results of such a model, but its development is outside the scope of the present paper. A consequence of the procedure followed here is that it is not possible to investigate the effect of changes in fuel prices or other taxes on the choice of car quality and on fixed and variable car costs.

The model we have estimated emphasizes the relationship between the demand for kilometers of the two cars. Indeed, the model can be interpreted as first determining the total demand for car kilometers, and subsequently distributing this total over the two cars, on the basis of the main driver's gender and the difference in variable costs between the two cars. The parameter that indicates the sensitivity of this distribution for the cost difference also indicates the substitution possibilities. Other household variables such as income and the number of workers did not appear to have a significant influence on this distribution.

We used the estimated model to simulate the effects of a shift of the burden of car taxes from fixed to variable costs and found, somewhat surprisingly, that such a shift will increase the demand for car kilometers. The higher variable costs have only a limited effect on the demand for kilometers, since the price elasticity is low (in absolute value). However, demand for second cars is highly sensitive to changes in disposable income and this means that the decrease in fixed car costs has a relatively large effect on the ownership of second cars. The increase in the demand for kilometers that results from the larger number of second cars outweighs the effect of the increase in variable costs.

\section{References}

Blackorby, C., D. Primont and R.R. Russell (1978) Duality, Separability and Functional Structure, American Elsevier, New York.

Deaton and Muellbauer (1980) Economics and Consumer Behavior, Cambridge University Press, Cambridge.

Espey, M. (1998) Gasoline Demand Revisited: An International Meta-Analysis of Elasticities Energy Economics 20 273-295.

Fisher, F.M. and K. Shell (1971) Taste and Quality Change in the Pure Theory of the Cost of Living Index, in: Z. Grilliches (ed.) Price Indexes and Quality Change, Harvard University Press, Cambridge (Ma), pp. 16-54.

Golob, T.F., S. Kim and W. Ren (1996) How Households Use Different Types of Vehicles: A Structural Driver Allocation and Usage Model Transportation Research 30A 103-118.

Golob, T.F. and M.G. McNally (1997) A Model of Activity Participation and Travel Interaction Between Household Heads Transportation Research 31B 177-194.

Graham, D.J. and S. Glaister (2002) The Demand for Automobile Fuel; A Survey of Elasticities Journal of Transport Economics and Policy 36 1-26.

Hensher, D.A. (1985) An Econometric Model of Vehicle Use in the Household Sector. Transportation Research, 19B, 303-313.

Jong, G.C. de (1990) An Indirect Utility Model of Car Ownership and Private Car Use European Economic Review 34 971-985.

Jong, G.C. de (1991) A Micro-Economic Model of the Joint Decision on Car Ownership and Car Use, in: Methods for Understanding Travel Behavior in the 1990s Proceedings, Conference International sur les Comportements de Deplacements, Quebec, pp. 387-403. 
Mannering, F.L. (1983) An Econometric Analysis of Vehicle Use in Multivehicle Households. Transportation Research, 17A, 183-189.

Mannering, F.L. and K. Train (1985) Recent Directions in Automobile Demand Modelling. Transportation Research, 19B, 265-274.

Mannering. F.L. and C. Winston (1985) A Dynamic Empirical Analysis of Household Vehicle Ownership and Utilization. RAND Journal of Economics, 16, 215-236.

Neary, J.P. and K.W.S. Roberts (1980) The Theory of Household Behaviour under Rationing European Economic Review, 13, 25-42.

Rouwendal, J. and F. de Vries (1999) The Taxation of Drivers and the Choice of Car Fuel Type Energy Economics 21 17-35.

Train, K. (1986) Qualitative Choice Analysis, MIT Press, Cambridge (Ma)

Train, K. and M. Lohrer (1983) Vehicle Ownership and Usage: An Integrated System of Disaggregate Demand Models, Paper presented at TRB Annual Meeting.

Verbeek, M. (2000) A Guide to Modern Econometrics, Wiley, Chichester. 